\title{
Diferencias de sexo en las estrategias cognitivas de regulación emocional: un reporte exploratorio en estudiantes universitarios
}

\section{Sex differences in cognitive emotion regulation strategies:} An exploratory report on college students

\author{
Sergio Dominguez-Lara* \\ Instituto de Investigación de la Escuela de Psicología, \\ Universidad de San Martín de Porres, Perú
}

\section{Resumen}

Se plantea como objetivo analizar las diferencias entre hombres y mujeres respecto a las estrategias cognitivas de regulación emocional en una muestra de 476 alumnos, con edades entre 16 y 32 años (78.4\% mujeres; $M_{\text {edad }}=20.57$ años), de una universidad privada de Lima. Se administró el Cognitive Emotional Regulation Questionarie - 18 y se implementó un análisis comparativo basado en medidas de magnitud del efecto. Los resultados muestran que las mujeres puntúan más alto en catastrofización y los hombres en aceptación. Se discuten las implicancias teóricas y prácticas de los hallazgos.

Palabras clave: estrategias cognitivas; regulación emocional; estudiantes universitarios; diferencias de sexo

\begin{abstract}
The objective of this study was to analyze the differences between men and women in terms of cognitive emotion regulation strategies in a sample of 476 students from 16 to 32 years ( $78.4 \%$ women, $\mathrm{M}_{\text {age }}=20.57$ years) from a private university in Lima. The Cognitive Emotion Regulation Questionnaire
\end{abstract}

Este es un artículo Open Access bajo la licencia Creative Commons Atribución-NoComercial-Compartirlgual 4.0

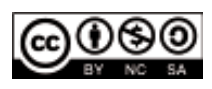


- 18 was administered and a comparative analysis based on effect size measures was implemented. The results show that women have higher scores in catastrophizing and men in acceptation. The theoretical and practical implications of the findings are discussed.

Keywords: cognitive strategies; emotion regulation; college students; sex differences

\section{Introducción}

Existen múltiples estresores en el ámbito universitario (exámenes, exposiciones, etc.), y las emociones negativas derivadas de esa experiencia (p. ej., culpa, vergüenza, cólera, etc.) necesitan ser reguladas de forma eficiente, ya que de lo contrario podrían derivar en ansiedad o depresión (Aldao, 2012; Dominguez-Lara, 2017).

Se conoce que en distintas latitudes existe mayor prevalencia de problemas de salud mental (p. ej., ansiedad, depresión) en las mujeres en comparación con los varones (Kessler, Chiu, Demler, \& Walters, 2005), y en Perú la situación es similar (Fiestas \& Piazza, 2014; Ministerio de Salud, 2011). No obstante, en población universitaria no existe una diferenciación respecto a la afectación de la persona tomando como variable diferencial el sexo, aun cuando la depresión y ansiedad son problemas frecuentes en este grupo (Lester, 2014; Lipson, Gaddis, Heinze, Beck, \& Eisenber, 2015; Vílchez-Cornejo et al., 2016).

Ante esta situación, un aspecto clave son las estrategias cognitivas de regulación emocional - ECRE (Garnefski, Kraaij, \& Spinhoven, 2002), que hacen referencia al abordaje cognitivo que realiza la persona de la información de tipo emocional que proviene del entorno, en su mayoría de eventos adversos (Garnefski \& Kraaij, 2007). Para la evaluación de esas estrategias fue creado el Cognitive Emotional Regulation Questionarie - CERQ (Garnefski et al., 2002), que cuenta con estudios instrumentales en diversos países, incluyendo el Perú (Dominguez-Lara \& Medrano, 2016; Dominguez-Lara \& Merino-Soto, 2015).

El modelo presenta varias estrategias diferenciadas que son utilizadas ante algún evento displacentero: Rumiación (pensar excesivamente sobre los 
sentimientos y pensamientos relacionados con el evento ocurrido), Catastrofización (tener pensamientos excesivos que enfatizan la situación experimentada), Autoculparse (la causa del evento displacentero se atribuye a la propia persona), Culpar a Otros (donde la causa del evento displacentero se atribuye a otras personas), Poner en Perspectiva (comparar la gravedad del evento con otros que se experimentaron anteriormente, a fin de disminuir el impacto), Aceptación (aceptar la ocurrencia que el evento displacentero ocurrió), Focalización positiva (tener pensamientos agradables y alegres, en lugar de focalizarse en el evento adverso), Reinterpretación positiva (involucra pensamientos que le dan un significado positivo al evento displacentero), y Refocalización en los planes (pensar en los pasos que deben llevarse a cabo para llegar a una solución para el problema que aqueja a la persona) (DominguezLara \& Merino-Soto, 2015). En diversos estudios se aprecia que los cuatro primeros tienden a asociarse a emociones negativas, malestar emocional, etc.; mientras que los últimos a emociones positivas, satisfacción, etc.; por lo que conocer en qué grado se manifiestan en las personas podría ayudar a predecir algunas conductas disfuncionales (Dominguez-Lara, 2017).

Algunas investigaciones han abordado las diferencias entre varones y mujeres respecto a las ECRE, encontrando que, frente a la ocurrencia de un evento adverso, las mujeres presentan mayor uso de las estrategias rumiación (Duarte, Matos, \& Marques, 2015; Martins, Freire, \& Ferreira-Santos, 2016), autoculpa (Chen, Yao, \& Xin, 2018; Duarte et al., 2015), poner en perspectiva (Medrano, Moretti, Ortiz, \& Pereno, 2013), refocalización en los planes, reinterpretación positiva (Chen et al., 2018); y en otros trabajos los varones destacan en autoculpa y culpar a otros (Martins et al., 2016). Finalmente, existe un estudio donde las diferencias entre varones y mujeres son tan pequeñas que no pueden considerarse significativas (Zhu et al., 2008).

Los resultados presentados previamente son interesantes porque plantearían la existencia de diferencias inherentes al sexo respecto a la forma de regular las emociones, y la neurociencia abordó este tópico con anterioridad. Por ejemplo, en algunos estudios se observó una mayor reactividad emocional en las mujeres (Gardener, Carr, MacGregor, \& Felmingham, 2013; McRae, Ochsner, Mauss, Gabrieli, \& Gross, 2008), y el uso de la reinterpretación (estrategia de regulación emocional) más eficiente y con menor esfuerzo en los varones para disminuir emociones negativas, mientras 
que en las mujeres el uso de esa estrategia se orienta a la transformación de las emociones negativas en positivas (McRae et al., 2008), aunque para aumentar la intensidad de una respuesta negativa (Gardener et al., 2013). Asimismo, el procesamiento cognitivo para regular emociones es más fuerte en hombres, y en las mujeres destaca el procesamiento afectivo (Mak, $\mathrm{Hu}$, Zhang, Xiao, \& Lee, 2009), aunque en otro estudio no hubo diferencias en cuanto al uso de estrategias de regulación emocional según el sexo (Domes et al., 2010).

Con todo, estas diferencias aun no fueron investigadas en Perú, por lo que es necesario aportar evidencia sobre la magnitud de las diferencias encontradas entre varones y mujeres en cuanto al uso de las ECRE. En ese sentido, el objetivo del presente reporte breve es determinar si existen diferencias respecto al sexo en cuanto al uso de las ECRE.

Es importante estudiar este aspecto porque en el ámbito universitario existen carreras profesionales donde son mayoritariamente mujeres (p. ej., enfermería, obstetricia, psicología, etc.) y, de acuerdo con lo mostrado, existiría mayor prevalencia de problemas de salud mental en aquel grupo, sobre todo si se trata de un contexto con altas demandas académicas y personales, y si se considera que las mujeres perciben mayor exigencia en entornos de educación superior que los varones (Dominguez-Lara \& Campos-Uscanga, 2017). Además, en vista de los costos que acarrean estos desórdenes, es necesario conocer aquellos factores que podrían predisponer a la persona a esas condiciones (Gustavsson et al., 2011).

\section{Método}

\section{Participantes}

Se evaluaron a 476 estudiantes de psicología que cursaban entre el tercer y séptimo ciclo en una universidad privada de Lima Metropolitana; 78.4\% mujeres; con edades entre 16 y 32 años $(M=20.57 ; D E=2.77) ; 93.9 \%$ de estado civil soltero; y $27.5 \%$ que trabaja. 


\section{Instrumento}

- Cognitive Emotional Regulation Questionarie - 18 (CERQ-18) (DominguezLara, \& Merino-Soto, 2015, 2018). La versión peruana tiene como base la versión extensa del CERQ (Garnefski et al., 2002). Consta de 18 ítems escalados en formato Likert con cinco opciones etiquetadas de respuesta, desde Casi nunca (1) hasta Casi siempre (5). Evalúa nueve estrategias cognitivas (dos ítems en cada una): Rumiación, Catastrofización, Autoculparse, Culpar a otros, Poner en perspectiva, Aceptación, Focalización Positiva, Reinterpretación positiva, y Focalización en los planes.

\section{Procedimiento}

Esta investigación forma parte de un proyecto mayor aprobado por la universidad del autor. Se solicitó el permiso respectivo a las instancias pertinentes. La administración de los instrumentos se realizó en horario de clase, lo que fue coordinado con los docentes de cada asignatura. Luego de firmar el consentimiento informado los estudiantes completaron los cuestionarios de manera voluntaria y anónima.

\section{Análisis de datos}

La aproximación de los datos a la normalidad se evaluó por medio de los estadísticos de dispersión (asimetría y curtosis; $<|1.5|$ ) y el índice estandarizado de asimetría: IEA < .25 (Malgady, 2007), previo al análisis comparativo.

El análisis principal se basó en la comparación entre hombres y mujeres. Fueron comparados en las estrategias evaluadas con el CERQ bajo un enfoque de magnitud del efecto con la $d$ de Cohen (1992): $\leq .20$, diferencia insignificante; entre .20 y .50 , pequeña; entre .50 y .80 , moderada; y $>.80$, grande. 


\section{Resultados}

\section{Análisis descriptivo}

La magnitud de la asimetría, curtosis y el IEA indican que existe una aproximación a la normalidad univariada.

\section{Análisis principal}

Se hallaron diferencias en catastrofización (mujeres > hombres) y aceptación (hombres $>$ mujeres), así como puntuaciones equivalentes en las demás estrategias (Tabla 1).

\section{Tabla 1}

Análisis descriptivo y comparativo de las estrategias cognitivas de regulación emocional

\begin{tabular}{|c|c|c|c|c|c|c|c|}
\hline & & \multicolumn{2}{|c|}{$\begin{array}{l}\text { Estadísticos } \\
\text { descriptivos }\end{array}$} & \multicolumn{2}{|c|}{$\begin{array}{l}\text { Estadísticos de } \\
\text { distribución }\end{array}$} & \multirow[b]{2}{*}{ IEA } & \multirow[b]{2}{*}{$d$} \\
\hline & & $M$ & $D E$ & $g_{1}$ & $g_{2}$ & & \\
\hline \multirow[t]{2}{*}{ Rumiación } & M & 5.83 & 1.733 & .185 & -.145 & .031 & .081 \\
\hline & $\mathrm{H}$ & 5.69 & 1.749 & .243 & -.675 & .040 & \\
\hline \multirow[t]{2}{*}{ Catastrofización } & M & 5.17 & 1.903 & .367 & -.265 & .051 & .244 \\
\hline & $\mathrm{H}$ & 4.70 & 2.019 & .699 & -.023 & .086 & \\
\hline \multirow[t]{2}{*}{ Auto-culparse } & M & 7.63 & 1.685 & -.588 & .083 & .104 & .059 \\
\hline & $\mathrm{H}$ & 7.73 & 1.789 & -.565 & -.453 & .088 & \\
\hline \multirow[t]{2}{*}{ Culpar a otros } & M & 3.22 & 1.490 & 1.095 & .543 & .247 & .094 \\
\hline & $\mathrm{H}$ & 3.36 & 1.461 & .717 & -.371 & .168 & \\
\hline \multirow[t]{2}{*}{ Poner en perspectiva } & M & 6.49 & 1.663 & -.094 & -.309 & .017 & .110 \\
\hline & $\mathrm{H}$ & 6.30 & 1.939 & -.074 & -.614 & .01 & \\
\hline \multirow[t]{2}{*}{ Aceptación } & M & 7.40 & 1.760 & -.387 & -.134 & .062 & .240 \\
\hline & $\mathrm{H}$ & 7.82 & 1.708 & -.405 & -.778 & .069 & \\
\hline \multirow[t]{2}{*}{ Focalización positiva } & M & 6.16 & 1.795 & -.016 & -.276 & .002 & .093 \\
\hline & $\mathrm{H}$ & 5.99 & 1.953 & -.091 & -.266 & .012 & \\
\hline \multirow[t]{2}{*}{ Reinterpretación positiva } & M & 6.72 & 1.912 & -.257 & -.301 & .035 & .068 \\
\hline & $\mathrm{H}$ & 6.59 & 1.958 & -.113 & -.544 & .015 & \\
\hline \multirow[t]{2}{*}{ Focalización en los planes } & M & 7.89 & 1.501 & -.555 & .354 & .123 & .040 \\
\hline & $\mathrm{H}$ & 7.83 & 1.485 & -.371 & -.125 & .084 & \\
\hline
\end{tabular}

Nota: $M=$ Media; $D E=$ Desviación estándar; $\mathrm{g}_{1}=$ asimetría; $\mathrm{g}_{2}=$ curtosis; IEA = índice estandarizado de asimetría; $d=d$ de Cohen; $\mathrm{H}=$ hombres; $\mathrm{M}=$ mujeres. 


\section{Discusión}

Se planteó como objetivo en la presente investigación explorar las diferencias de sexo respecto a las ECRE en estudiantes universitarios, considerando los trabajos previos y la necesidad de profundizar este tema que no cuenta con estudios en el Perú.

La evidencia previa indica que si bien las mujeres usan con mayor frecuencia algunas ECRE asociadas al malestar (Chen et al., 2018; Duarte et al., 2015; Martins et al., 2016), también lo hacen en las relacionadas al bienestar (Chen et al., 2018), lo que se vincula con un procesamiento tanto afectivo como cognitivo, y no predominantemente afectivo como se ha señalado en otras investigaciones (Mak et al., 2009). En ese sentido, los hallazgos del presente estudio están en la senda de los que concluyen que no existen diferencias marcadas entre un grupo u otro (Domes et al., 2010; Zhu et al, 2008).

Sin embargo, es probable que la reactividad emocional vinculada en mayor grado al sexo femenino (Gardener et al., 2013; McRae et al., 2008) se relacione con el uso de la estrategia catastrofización por las estudiantes que participaron de la investigación, lo que podrían incrementar la intensidad de la respuesta emocional negativa derivada de la experiencia displacentera. Esto es relevante porque la catastrofización se constituye como un factor de riesgo para la ansiedad y depresión (Dominguez-Lara, 2017), y si bien el panorama indica que existe un equilibrio entre grupos respecto al uso de las ECRE, estos hallazgos puntuales pueden servir para orientar las consejerías en este contexto.

Con relación a la aceptación, es un hallazgo inédito que sea usado en mayor medida por hombres, y a su vez refuerza los planteamientos del procesamiento cognitivo en este grupo (Mak et al., 2009), porque la aceptación se refiere a la toma de conciencia de que los estados emocionales negativos son inevitables (Dominguez-Lara \& Medrano, 2016).

Con todo, han podido identificarse algunas limitaciones. Si bien el tamaño muestral global no puede considerarse bajo, el desbalance en cuanto a la cantidad de hombres y mujeres, no permitió un análisis de invarianza de medición debido a problemas de identificación del modelo. Por otro lado, la 
evaluación de estudiantes de una sola carrera podría sesgar las interpretaciones, además de que sus respuestas pudieron ser afectadas por la deseabilidad social, teniendo en cuenta que son estudiantes de psicología y algunos ítems reflejan creencias que no serían compatibles con el perfil de la carrera profesional.

En vista de los resultados, se concluye que no existen diferencias en el uso de las ECRE entre varones y mujeres, excepto en catastrofización y aceptación.

Se recomienda replicar el estudio con un mayor número de participantes, y de distintas carreras, desde un enfoque de invarianza de medición, con la finalidad de poder explorar a profundidad aquellas diferencias asociadas al sexo desde diferentes niveles de medición del constructo. Asimismo, sería interesante explorar si la influencia de las ECRE sobre variables afectivas (p. ej., ansiedad) difiere según el sexo.

\section{Referencias}

Aldao, A. (2012). Emotion regulation strategies as transdiagnostic processes: a closer look at the invariance of their form and function. Revista de Psicopatología y Psicología Clínica, 17(3), 261-277.

Chen, Y. N., Yao, S. Q., \& Xin, X. H. (2018). Cognitive emotion regulation strategies among Chinese adolescents having unprotected sex: a latent profile analysis. Psychology, Health \& Medicine, 23(1), 58-68.

Cohen, J. (1992). A power primer. Psychological Bulletin, 112(1), 155-159.

Domes, G., Schulze, L., Böttger, M., Grossmann, A., Hauenstein, K., Wirtz, P. H., ... Herpertz, S. C. (2010). The neural correlates of sex differences in emotional reactivity and emotion regulation. Human brain mapping, 31(5), 758-769.

Dominguez-Lara, S. (2017). Influencia de las estrategias cognitivas de regulación emocional sobre la ansiedad y depresión en universitarios: análisis preliminar. Salud Uninorte, 33(3), 315-321.

Dominguez-Lara, S., \& Campos-Uscanga, Y. (2017). Influencia de la satisfacción con los estudios sobre la procrastinación académica en estudiantes de psicología: Un estudio preliminar. Liberabit, 23(1), 123-135.

Dominguez-Lara, S., \& Medrano, L. (2016). Propiedades psicométricas del Cognitive Emotional Regulation Questionnaire (CERQ) en estudiantes universitarios de Lima. Psychologia: Avances en la disciplina, 10(1), 53-67.

Dominguez-Lara, S., \& Merino-Soto, C. (2015). Una versión breve del Cognitive Emotional Regulation Questionnaire: Análisis estructural del CERQ-18 en estudiantes universitarios limeños. Revista Peruana de Psicología y Trabajo Social, 4(1), 25-36.

Dominguez-Lara, S., \& Merino-Soto, C. (2018). Cognitive Emotional Regulation Questionnaire-18 en universitarios: evidencias de validez convergente y discriminante. Revista Iberoamericana de Diagnóstico y Evaluación Psicológica-E Avaliação Psicológica, 47(2), 171-184. 
Duarte, A. C., Matos, A. P., \& Marques, C. (2015). Cognitive emotion regulation estrategias and depressive symptoms: gender's moderating effect. Procedia-Social and Behavioral Sciences, 165, 275-283.

Fiestas, F., \& Piazza, M. (2014). Prevalencia de vida y edad de inicio de transtornos mentales en el Perú urbano: Resultados del estudio mundial de salud mental, 2005. Revista Peruana de Medicina Experimental y Salud Pública, 31(1), 39-47.

Gardener, E. K. T., Carr, A. R., MacGregor, A., \& Felmingham, K. L. (2013). Sex differences and emotion regulation: An event-related potential study. PLOS ONE, 8(10), e73475.

Garnefski, N., \& Kraaij, V. (2007). The Cognitive Emotion Regulation Questionnaire: Psychometric features and prospective relationships with depression and anxiety in adults. European Journal of Psychological Assessment, 23(3), 141-149.

Garnefski, N., Kraaij, V., \& Spinhoven, P. (2002). Manual for the use of Cognitive Emotion Regulation Questionnaire. Leiderdorp, the Netherlands: DATEC.

Gustavsson, A., Svensson, M., Jacobi, F., Allgulander, C., Alonso, J., Beghi, E., ... Gannon, B. (2011). Cost of disorders of the brain in Europe 2010. European neuropsychopharmacology, 21(10), 718-779.

Kessler, R. C., Chiu, W. T., Demler, O., \& Walters, E. E. (2005). Prevalence, severity, and comorbidity of 12month DSM-IV disorders in the National Comorbidity Survey Replication. Archives of General Psychiatry, 62(6), 617-627.

Lester, D. (2014). College student stressors, depression, and suicidal ideation. Psychological Reports, 114(1), 293-296.

Lipson, S. K., Gaddis, S. M., Heinze, J., Beck, K., \& Eisenber, D. (2015). Variations in student mental health and treatment utilization across US colleges and universities. Journal of American College Health, 63(6), 388396.

Mak, A. K. Y., Hu, Z., Zhang, J. X. X., Xiao, Z., \& Lee, T. M. C. (2009). Sex-related differences in neural activity during emotion regulation. Neurpsychologia, 47, 2900-2908.

Malgady, R. (2007). How skew are psychological data? A standardized index of effect size. The Journal of General Psychology, 134(3), 355-359.

Martins, E. C., Freire, M., \& Ferreira-Santos, F. (2016). Examination of adaptive and maladaptive cognitive emotion regulation strategies as transdiagnostic processes: associations with diverse psychological symptoms in college students. Studia Psychologica, 58(1), 59-73.

McRae, K., Ochsner, K. N., Mauss, I. B., Gabrieli, J. J., \& Gross, J. J. (2008). Gender differences in emotion regulation: An fMRI study of cognitive reappraisal. Group processes \& intergroup relations, 11(2), 143-162.

Medrano, L., Moretti, L., Ortiz, A., \& Pereno, G. (2013). Validación del Cuestionario de Regulación Emocional Cognitiva en Universitarios de Córdoba, Argentina. Psykhe, 22(1), 83-96.

Ministerio de Salud [MINSA]. (2011). Análisis de Situación de Salud de las y los Jóvenes. «Una mirada al Bono Demográfico». Recuperado de http://bvs.minsa.gob.pe/local/minsa/2284.pdf

Vílchez-Cornejo, J., Quiñones-Laveriano, D., Failoc-Rojas, V., Acevedo-Villar, T., Larico-Calla, G., MucchingToscano, S., ... Díaz-Vélez, C. (2016). Salud mental y calidad de sueño en estudiantes de ocho facultades de medicina humana del Perú. Revista Chilena de Neuro-psiquiatría, 54(4), 272-281.

Zhu, X., Auerbach, R., Yao, S., Abela, J., Xiao, J., \& Tong, X. (2008). Psychometric properties of the Cognitive Emotion Regulation Questionnaire: Chinese version. Cognition and Emotion, 22(2), 288-307. 